\title{
Pushing radiation therapy limitations with theranostic nanoparticles
}

\author{
"Nanoparticles design is a rising landscape \\ in the era of modern oncology offering new \\ perspectives."
}

First draft submitted: 2 March 2016; Accepted for publication: 10 March 2016; Published online: 13 April 2016

Keywords: clinical trial • gadolinium • image-guided radiation therapy • radiosensitizer - theranostic nanoparticles

In radiation oncology, while radiation is effective in killing cancer cells, the dose safely deliverable to the target volume is often limited by the possibility of collateral damage to surrounding healthy tissues. However, for some cancer sites, it has been shown that a dose escalation in the tumor could significantly improve local control and patient survival [1]. While recently developed methods such as intensity-modulated radiation therapy, volumetric-modulated arc therapy and imageguided radiation therapy have improved the delivered radiation dose conformality, dose escalation remains an important clinical challenge that needs to be addressed to improve the efficacy of radiation therapy [2].

Nanoparticles design is a rising landscape in the era of modern oncology offering new perspectives. The enhancement of radiation effect induced by radiosensitizing nanoparticles is probably one of the most translational aspects. Radiosensitizing nanoparticles increase the radiobiological effects within the site of disease while maintaining the current clinical constraints on dose delivered to healthy organs. The photoelectric interaction increases strongly as a function of the atomic number of the nanoparticle (proportional to $Z^{4}-Z^{5}$ ), giving these particles a high interaction probability with low-energy photons and allowing the formation of additional diffused photons, photoelectrons, Auger electrons and reactive oxygen species that have the potential to amplify the biological damage. Even under high-energy photon irradiation, high$\mathrm{Z}$ nanoparticles can interact with primary or secondary species permitting a highly efficient nanoscale dose deposition around the nanoparticle [3]. Consequently, the differential effect between healthy tissue and tumor tissue is improved.

The efficacy of nanoparticles as radiosensitizers has been demonstrated at the preclinical level for multiple high- $Z$ nanoparticles since Hainfeld et al. demonstrated the potential of gold nanoparticles after systemic injection [4], and at the clinical level for hafnium nanoparticles [5]. However, because of the injection method (intratumoral injection) and because of their size $(50 \mathrm{~nm})$, the hafnium nanoparticle applications are mainly limited to easyto-access tumor sites, such as sarcoma, head and neck and prostate cancers. For the majority of other cancer sites, intravenous injection remains an obligation. To address these limitations while still ensuring low toxicity to the healthy tissues, theranostic nanoparticles were created, combining diagnostic and therapeutic properties.

Gadolinium is the most well-known element for biomedical applications. Its use in medicine is directly correlated with the development of contrast agents for MRI, a noninvasive and nonionizing method for acquiring volumetric images with high spatial resolution and excellent soft-tissue contrast. Some clinics have begun to replace CT simulation with MRI simulation for radiation treatment
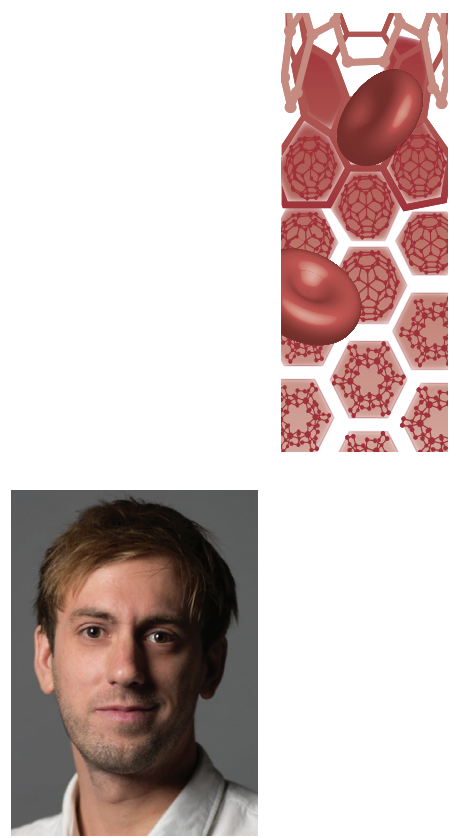

Alexandre Detappe Institut Lumiere Matiere, UMR 5306 CNRS, Lyon 1 University, Lyon, France and

Department of Radiation Oncology, Dana-Farber Cancer Institute, Harvard Medical School, Boston, MA 02215, USA

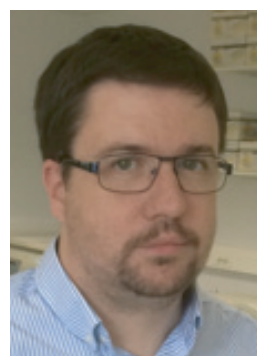

Francois Lux

Institut Lumiere Matiere, UMR 5306 CNRS, Lyon 1 University, Lyon, France

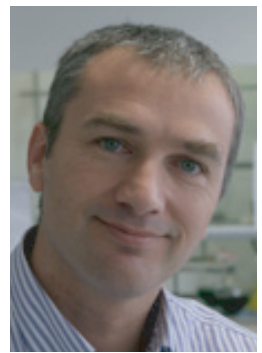

Olivier Tillement Author for correspondence: Institut Lumiere Matiere, UMR 5306 CNRS, Lyon 1 University, Lyon, France olivier.tillement@univ-lyon1.fr

Future $\because$ Medicine part of 
planning purposes, and future MR-LINAC and MRbased real-time tumor tracking techniques are under development $[6,7]$. Besides their use for imaging applications, gadolinium-based compounds are also envisaged for therapeutic applications. Gadolinium displays high capture cross-section due to its relatively high atomic number $(Z=64)$ and can interact with high energies irradiations, making it an effective radiosensitizer.

\section{"The enhancement of radiation effect induced by radiosensitizing nanoparticles is probably one of the most translational aspects.}

Our group developed AGuIX (Activation and Guidance of irradiation by $\mathrm{x}$-ray), a sub-5 $\mathrm{nm}$ nanoparticle based on a polysiloxane network surrounded by gadolinium chelates [8,9], designed to be an effective MRI contrast agent and radiosensitizer. Because of the VEGF produced by tumor cells to stimulate the tumor angiogenesis and of the lack of effective lymphatic drainage in the tumor tissues, AGuIX can penetrate the tumor after a systemic injection thanks to the enhanced permeability and retention effect. The nanoparticles stay inside the tumor for a significant period of time, before being cleared by the kidneys, avoiding toxicity issues by limiting their exposition to healthy organs. Preclinical studies conducted in various tumor models have demonstrated that low concentrations of AGuIX injected intravenously were attractive radiosensitizers with a significant therapeutic effect, while confirming that the nanoparticle is nontoxic [10]. In addition, its high longitudinal relaxivity allows for better contrast properties than current US FDA-approved gadolinium chelates, creating a potential for future use in MRI-

\section{References}

1 Budiharto T, Haustermans K, Van Cutsem E et al. A Phase I radiation dose-escalation study to determine the maximal dose of radiotherapy in combination with weekly gemcitabine in patients with locally advanced pancreatic adenocarcinoma. Radiat. Oncol. 3, 30 (2008).

2 Jaffray DA. Image-guided radiotherapy: from current concept to future perspectives. Nat. Rev. Clin. Oncol. 9(12), 688-699 (2012).

3 Mcquaid HN, Muir MF, Taggart LE et al. Imaging and radiation effects of gold nanoparticles in tumour cells. Sci. Rep. 6, 19442 (2016).

4 Hainfeld JF, Slatkin DN, Smilowitz HM. The use of gold nanoparticles to enhance radiotherapy in mice. Phys. Med. Biol. 49(18), N309-N315 (2004).

5 Min Y, Caster JM, Eblan MJ, Wang AZ. Clinical translation of nanomedicine. Chem. Rev. 115(19), 1114711190 (2015). guiding radiation therapy, notably with the upcoming MR-LINAC technology [11,12].

The logical step after demonstrating the potential of AGuIX at the preclinical level for several tumor localizations is to continue the nanoparticles development to the clinical step. A current GMP manufacturing process has been developed and the regulatory toxicity tests on two animal models (rodents and monkeys) have shown no evidence of toxicity. A clinical Phase I will be investigated in France by our group in close collaboration with NH TherAguix SAS in order to determine the safety of AGuIX and its maximum tolerated dose in patients concurrently treated with standard chemoradiation; define the recommended phase II dose of AGuIX; and define the biodistribution of a single intravenous injection as well as multiple AGuIX injections in order to provide a rationale of AGuIX dosing administration. These results will demonstrate the translationality of using nanoparticles as image-guided radiation therapy agents and further clinical trial will be performed to quantify the therapeutic benefits of AGuIX. The proof-of-concept of using AGuIX as a theranostic agent in human will be a breakthrough approach for patient benefit.

\section{Financial \& competing interests disclosure}

F Lux, and O Tillement have one patent to disclose: WO2011135101. This patent protects some of the nanoparticles described in this publication: AGulX. The authors have no other relevant affiliations or financial involvement with any organization or entity with a financial interest in or financial conflict with the subject matter or materials discussed in the manuscript apart from those disclosed.

No writing assistance was utilized in the production of this manuscript.

6 (P015) Radiation Biological Responses of MRI-Linac vs Linac in Human Head and Neck and Lung Cancer Cells. Oncology 29(4 Suppl. 1), pii:205024 (2015) (Epub ahead of print).

7 Keall PJ, Barton M, Crozier S et al. The Australian magnetic resonance imaging-linac program. Semin. Radiat. Oncol. 24(3), 203-206 (2014).

8 Sancey L, Lux F, Kotb S et al. The use of theranostic gadolinium-based nanoprobes to improve radiotherapy efficacy. Br. J. Radiol. 87(1041), 20140134 (2014).

9 Lux F, Sancey L, Bianchi A et al. Gadoliniumbased nanoparticles for theranostic MRIradiosensitization. Nanomedicine (Lond.) 10(11), 1801-1815 (2015).

10 Sancey L, Kotb S, Truillet C et al. Long-term in vivo clearance of gadolinium-based AGuIX nanoparticles and their biocompatibility after systemic injection. ACS Nano 9(3), 2477-2488 (2015). 
11 Detappe A, Kunjachan S, Rottmann J et al. AGuIX nanoparticles as a promising platform for image-guided radiation therapy. Cancer Nanotechnol. 6(1), 4 (2015).
12 Le Duc G, Miladi I, Alric C et al. Toward an image-guided microbeam radiation therapy using gadolinium-based nanoparticles. ACS Nano 5(12), 9566-9574 (2011). 